\title{
THE SOFT X-RAY EXCESSES OBSERVED WITH ROSAT
}

\author{
R. WALTER, H.H. FINK \\ Max Planck Inst. für extraterrestr. Physik, Giessenbachstraße, D-85748 Garching b. München
}

\begin{abstract}
The properties of the soft X-ray excesses of bright Seyfert 1 galaxies and Quasars are described using the observations obtained with the PSPC (0.1-2.4 keV) detector of the XRT telescope aboard ROSAT during the ROSAT all sky survey (RASS). The sample consists of 58 Seyfert 1 type AGN detected with more than 300 counts during the RASS and observed at least once with IUE.

The soft $\mathrm{X}$-ray photon indices of our sample members range from 1.6 to 3.4 in a wide distribution $(\langle\Gamma\rangle=2.50, \sigma=0.48)$. The width of the distribution is considerably larger than the mean statistical uncertainty on the individual spectral slopes $(\sigma=0.33)$. Excepting for IC 4329A and Mrk 766, the mean contribution of absorbing cold matter intrinsic to the Seyfert galaxies of our sample to the absorbing column density is less than $10^{20} \mathrm{~cm}^{-2}$. In IC $4329 \mathrm{~A}$ and Mrk 766 intrinsic absorbtion is observed at soft $\mathrm{X}$-ray. Both sources are also strongly reddened by dust.

An excess of soft $X$-ray flux is detected in $90 \%$ of the sources above the exptrapolation of the hard X-ray power law. It can be shown that the PSPC spectral slope is a measure of the strength of the soft $\mathrm{X}$-ray excess. If the reddened sources are excluded, a correlation appears between the strength of the ultraviolet blue bump and the soft $\mathrm{X}$-ray photon index (figure 1). The ratio of the ultraviolet to infrared fluxes and the ultraviolet spectral slope are also related to the strength of the blue bump. The observations are compatible with a model where most of the spectral variations arising among the sources studied are driven by the strength of the bump component, which varies by a factor of 100 from object to object. A bump model consisting of a power law with a high energy cutoff at $80 \mathrm{eV}$ can fit most of the sources. In any case, the spectral energy distribution of the ultraviolet to soft $\mathrm{X}$-ray bump is characterised by $\nu F_{\nu(1375 \AA)}=(1-5) \int_{\epsilon>150 e V} F_{\epsilon} d \epsilon$.
\end{abstract}

Further reading : R. Walter, H. H. Fink, 1993, A\&A 274, 105.

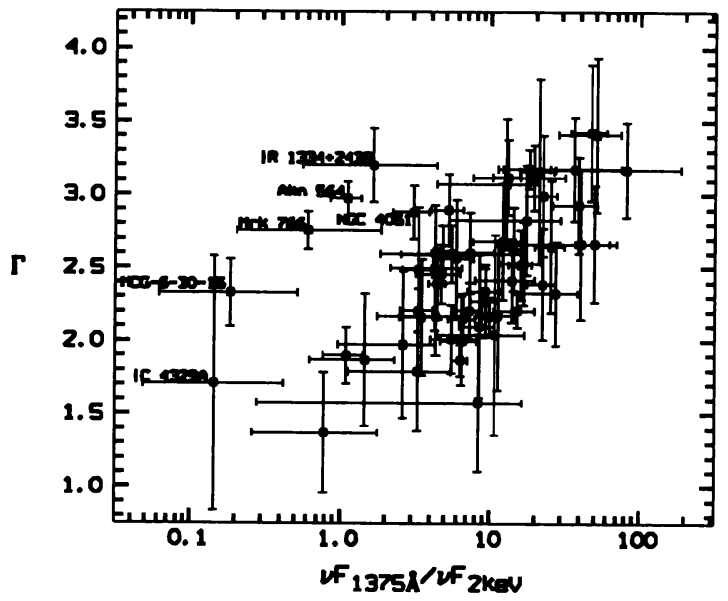

Fig. 1. Relation between the spectral slope $\Gamma$ measured in the PSPC band and the logarithm of the ratio of the ultraviolet $(1375 \AA)$ and the $2 \mathrm{keV}$ flux. Named sources are reddened.

338

T. J.-L. Courvoisier and A. Blecha: Multi-Wavelength Continuum Emission of AGN, 338.

(C) 1994 IAU. Printed in the Netherlands. 